\title{
Lifetime cost-effectiveness analysis of ticagrelor in patients with acute coronary syndromes based on the PLATO trial: a Singapore healthcare perspective
}

\author{
Chee Tang $\underline{\text { Chin }}^{1,2}$, MBChB, MRCP, Carl Mellstrom ${ }^{3}$, PhL, Terrance Siang Jin Chua ${ }^{1,2}$, MBBS,
} David Bruce Matchar $^{2}$, MD, FACP

INTRODUCTION Ticagrelor is a novel antiplatelet drug developed to reduce atherothrombosis. The PLATO trial compared ticagrelor and aspirin to clopidogrel and aspirin in patients with acute coronary syndromes (ACS). Ticagrelor was found to be superior in the primary composite endpoint of cardiovascular death, myocardial infarction or stroke, without increasing major bleeding events. The current study estimates the lifetime cost-effectiveness of ticagrelor relative to generic clopidogrel from a Singapore public healthcare perspective.

METHODS This study used a two-part cost-effectiveness model. The first part was a 12-month decision tree (using PLATO trial data) to estimate the rates of major cardiovascular events, healthcare costs and health-related quality of life. The second part was a Markov model estimating lifetime quality-adjusted survival and costs conditional on events during the initial 12 months. Daily drug costs applied were SGD 1.05 (generic clopidogrel) and SGD 6.00 (ticagrelor). Cost per quality-adjusted life years (QALY) was estimated from a Singapore public healthcare perspective using life tables and short-term costs from Singapore, and long-term costs from South Korea. Deterministic and probabilistic sensitivity analyses were performed.

RESULTS Ticagrelor was associated with a lifetime QALY gain of 0.13 , primarily driven by lower mortality. The resulting incremental cost per QALY gained was SGD 10,136.00. Probabilistic sensitivity analysis indicated that ticagrelor had a $>99 \%$ probability of being cost-effective, given the lower recommended WHO willingness-to-pay threshold of one GDP/capita per QALY.

CONCLUSION Based on PLATO trial data, one-year treatment with ticagrelor versus generic clopidogrel in patients with ACS, relative to WHO reference standards, is cost-effective from a Singapore public healthcare perspective.

Keywords: acute coronary syndromes, clopidogrel, cost-effectiveness, Singapore, ticagrelor

\section{INTRODUCTION}

Ischaemic heart disease, including acute coronary syndromes (ACS), is one of the leading causes of death and morbidity in Singapore. ${ }^{(1)}$ It was previously reported that in Singapore, the Global Registry of Acute Coronary Events in-hospital mortality score, derived from populations outside Asia, underestimated in-hospital mortality from ACS, even with the widespread adoption of evidence-based medications and treatments. ${ }^{(2)}$ The combination of aspirin and clopidogrel, as recommended by international practice guidelines, is currently the most widely used antiplatelet strategy for secondary prevention of atherothrombotic events among patients with ACS in Singapore. ${ }^{(3-6)}$

Ticagrelor, a novel reversibly binding and direct-acting oral antagonist of the platelet adenosine diphosphate receptor $\mathrm{P}_{2} \mathrm{Y}_{12}$, has been shown to yield faster, greater and more consistent $\mathrm{P}^{2} \mathrm{Y}_{12}$ inhibition than clopidogrel. ${ }^{(7)}$ In the PLATelet Inhibition and Patient Outcomes (PLATO) study, patients with ACS who were randomised to receive ticagrelor for 12 months showed a significant reduction in the combined endpoint of cardiovascular death, myocardial infarction (MI) or stroke, as compared to patients receiving clopidogrel, including a significant reduction in the individual endpoint of cardiovascular death without a significant difference in overall major bleeding. ${ }^{(8,9)}$ Based on this, the combination of aspirin and ticagrelor is now recommended in international treatment guidelines and is approved in Singapore for the treatment of patients with ACS. ${ }^{(3,5,6)}$

However, it is expected that the drug cost of ticagrelor would be greater than that of generic clopidogrel, therefore raising the issue of the cost-effectiveness of this new strategy of treating patients with ACS. A published cost-effectiveness analysis based on the PLATO results combined with Swedish unit costs and life tables concluded that within that context, treatment with ticagrelor for 12 months was associated with increased healthcare costs of $€ 362$ and a quality adjusted life year (QALY) gain of 0.13 compared with generic clopidogrel, yielding a cost per QALY gained with ticagrelor of $€ 2,753$. $^{(10)}$

No such data is currently available for countries in Asia and, specifically, Singapore. Therefore, we sought to build upon previous studies in order to determine the anticipated cost per QALY for ticagrelor and compare it to generic clopidogrel in the Singapore healthcare setting.

${ }^{1}$ National Heart Centre Singapore, ${ }^{2}$ Duke-NUS Graduate Medical School, Singapore, ${ }^{3}$ AstraZeneca R\&D, Sweden

Correspondence: Dr Chin Chee Tang, Consultant, Department of Cardiology, National Heart Centre Singapore, Mistri Wing, 17 Third Hospital Avenue, Singapore 168752.chin.chee.tang@nhcs.com.sg 


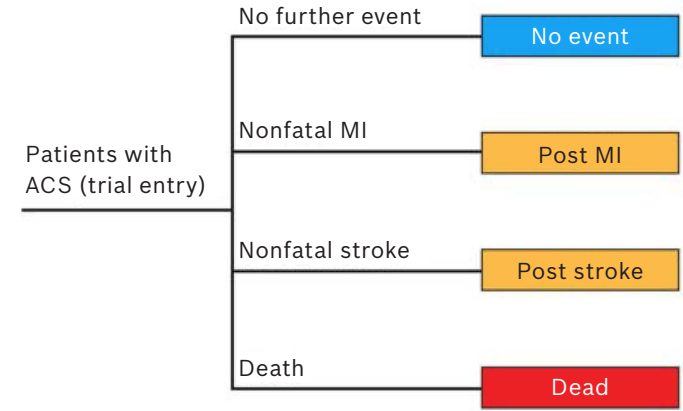

One-year decision tree

based on PLATO data
Start state

in long-term Markov model

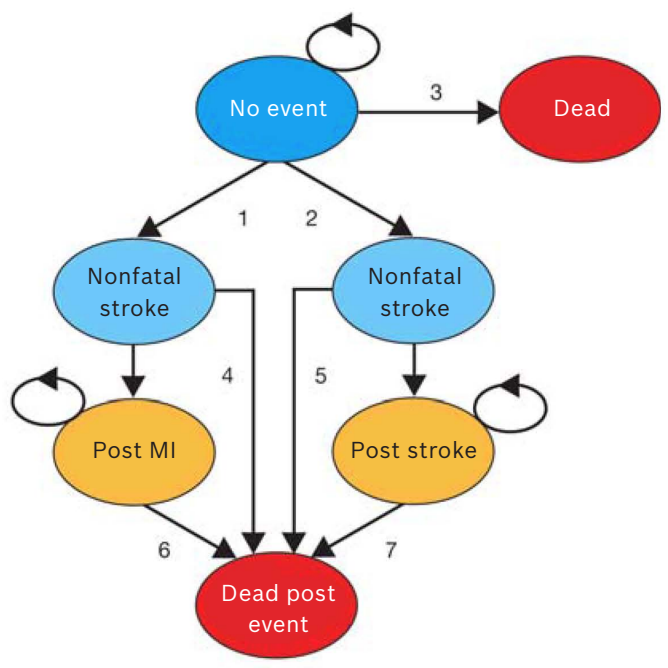

Long-term Markov extrapolation model

Fig. 1 Model structure consists of two parts. The first part is a simple decision tree representing the four primary outcomes of the PLATO trial over the one-year trial period: no event, nonfatal myocardial infarction (MI), nonfatal stroke or death. The second part is a Markov model, which simulates outcomes after the first year. As denoted by the numbers, individuals can remain event-free until they have an MI (1), a stroke (2), or die without a stroke or MI event (3); if they incur an event, they can experience an early death (4 or 5 ), or late death (6 or 7). This model structure was developed by Nikolic et al, and used with permission from European Heart Journal.(10) ACS: acute coronary syndromes

\section{METHODS}

The analysis was performed using a two-component decision model (Fig. 1) developed by Nikolic et al. ${ }^{(10)}$ The first component consisted of a simple tree corresponding to the period of the PLATO study (one-year). Subsequent events were modelled as a Markov structure with the potential for a recurrent event (MI or stroke) that could be fatal, or death from other causes. Model parameters corresponding to the first year following treatment are shown in Table I, and those for subsequent years are in Table II. ${ }^{(10-16)}$

Both clinical outcomes and resource counts were based on data from the PLATO study. ${ }^{(10)}$ Utility values during the trial period were used to create a weighted survival, i.e. quality-adjusted survival to one-year. Resource counts, including hospital stay, procedures, devices and blood products, were multiplied at the individual level by Singapore-specific costs from the public hospital cost accounting system. For the subsequent years, mortality rates were based on Singapore-specific general population life tables, ${ }^{(17)}$ inflated by a hazard ratio relevant to the nature of the state (no event, MI or stroke) and time since the event (first year or second and subsequent years). These hazard ratios were derived from PLATO data and published references. ${ }^{(11,12,13)}$

Costs for both hospital and outpatient care were assigned to each state based on time since entering the state (first year or second and subsequent years). As only reliable estimates for Singapore costs were available for hospital care, cost estimates were derived in two steps. First, hospital costs for each public hospital in Singapore were obtained from the public hospital accounting system, for diagnosis-related group codes corresponding to stroke and MI. The average stroke and MI costs were obtained by weighting of the number of patients in each corresponding code group. Second, data was obtained from
Table I. Model input parameters - initial one-year model.

\begin{tabular}{lll}
\hline Parameter & Ticagrelor & Clopidogrel \\
\hline Probability & & \\
All-cause death & 0.046 & 0.059 \\
Nonfatal MI & 0.050 & 0.058 \\
Nonfatal stroke & 0.010 & 0.009 \\
No event & 0.894 & 0.874 \\
Cost (SGD) & & \\
All-cause death & 20,018 & 20,454 \\
MI & 27,752 & 28,188 \\
Stroke & 23,907 & 24,344 \\
No event & 14,715 & 15,152 \\
Ticagrelor/day & 6.00 & $\mathrm{NA}$ \\
Clopidogrel/day & $\mathrm{NA}$ & 1.05 \\
QALY & & \\
All-cause death & 0.247 & 0.250 \\
MI & 0.811 & 0.814 \\
Stroke & 0.735 & 0.738 \\
No event & 0.873 & 0.876 \\
\hline
\end{tabular}

Source: PLATO data ${ }^{(10)}$

QALY: quality adjusted life years; MI: myocardial infarction; NA: not applicable

a South Korean report for hospital and outpatient costs in the first year, and second and subsequent years following a stroke and MI. ${ }^{(15)}$ These costs were divided by the Korean hospital costs for the first year to obtain costs that were relative to acute hospitalisation. The resulting ratios were multiplied by the Singapore acute hospital costs to obtain an imputed cost for hospital and outpatient costs in the first and subsequent years. The hospital and outpatient costs were summed to obtain the total cost for $\mathrm{Ml}$ and stroke for the first and subsequent years. As there is no reliable cost reference for individuals who remain alive without an event, we therefore applied the same cost as that for post-event MI.

Several assumptions were made to simplify model construction and parameter estimation. First, no more than one 
Table II. Model input parameters - Markov model inputs.

\begin{tabular}{|c|c|c|}
\hline Parameter & Baseline value & Source \\
\hline \multicolumn{3}{|l|}{ Annual risk } \\
\hline MI in no-event state & 0.019 & PLATO data ${ }^{(10)}$ \\
\hline Stroke in no-event state & 0.003 & PLATO data ${ }^{(10)}$ \\
\hline \multicolumn{3}{|l|}{ Increased risk of death* } \\
\hline No event state & 2.00 & Norhammar et $\mathrm{al}^{(11)}$ \\
\hline Nonfatal MI state & 6.00 & PLATO data ${ }^{(10)}$ \\
\hline Post-MI state & 3.00 & Assumption \\
\hline Nonfatal stroke state & 7.43 & Dennis et $\mathrm{al}^{(12)}$ \\
\hline Post-stroke state & 3.00 & Dennis et $\mathrm{al}^{(12)}$; Olai et $\mathrm{al}^{(13)}$ \\
\hline \multicolumn{3}{|l|}{ Annual cost (SGD) } \\
\hline No event state & 783 & Singapore public hospital \\
\hline Nonfatal MI state & 6,929 & accounting data ${ }^{(14)} ;$ plus \\
\hline Post-MI state & 783 & relative long-term \\
\hline Nonfatal stroke state & 9,999 & outpatient costs from \\
\hline Post-stroke state & $2,161 \mathrm{~J}$ & South Korea ${ }^{(15)}$ \\
\hline \multicolumn{3}{|c|}{ Annual QALY weight in the no event state } \\
\hline Age $<69$ yrs & 0.8748 & PLATO data ${ }^{(10)}$ \\
\hline Age $70-79$ yrs & 0.8430 & Burström and Rehnberg ${ }^{(16)}$ \\
\hline Age $>79$ yrs & 0.7814 & Burström and Rehnberg ${ }^{(16)}$ \\
\hline \multicolumn{3}{|l|}{ Annual QALY decrement } \\
\hline Nonfatal Ml state & 0.0627 & PLATO data ${ }^{(10)}$ \\
\hline Post-Ml state & 0.0627 & PLATO data ${ }^{(10)}$ \\
\hline Nonfatal stroke state & 0.1384 & PLATO data ${ }^{(10)}$ \\
\hline Post-stroke state & 0.1384 & PLATO data ${ }^{(10)}$ \\
\hline
\end{tabular}

*Hazard ratio over standard mortality.

MI: myocardial infarction; QALY: quality adjusted life years

MI or stroke event can occur. This assumption may diminish the impact in people who have multiple (and potentially progressively worse) events. To accommodate this, it was assumed that individuals who experienced an Ml or stroke after initiating treatment would have decreased utility. Second, using Korean outpatient vs. inpatient costs to estimate Singapore outpatient costs presumes that the patterns of outpatient care relative to hospital care are comparable in the two countries. To test the impact of this assumption, deterministic sensitivity analyses were performed by doubling and halving long-term costs to assess the difference in relative costs. Third, while study drug utilisation was lower than prescribed utilisation, for the purpose of calculating the incremental cost-effectiveness ratio (ICER), it was assumed that future patients would use medication as prescribed. This is a conservative assumption (because it introduces a bias against ticagrelor), as effectiveness was estimated on the basis of actual study drug use.

Applying the above estimates, a base case estimate of ICER was calculated by dividing the additional (incremental) lifetime cost of using ticagrelor per subject vs. clopidogrel by the incremental effectiveness of ticagrelor in terms of lifetime QALYs. QALYs are a standard metric that combines length of life and quality of life to approximate lifetime utility. ${ }^{(18)}$ To accommodate uncertainty in model inputs and to assess the robustness of base case results, two forms of sensitivity analysis were used. First, each input parameter was varied over a plausible range and the corresponding ICERs were calculated. Second, a probabilistic analysis using the bootstrap method was performed. To do this, the analysis described in the base case was repeated 10,000 times; with each repetition, input values were drawn from a stochastic model that incorporates residual uncertainty from a regression model of the PLATO data (for the short-term inputs) and from log normal and gamma distributions for the Markov model (for the long-term inputs). The latter analysis was presented with the results of each of the 10,000 simulations plotted on the incremental cost/incremental effectiveness plane, as well as a cost-effectiveness acceptability curve. A cost-effectiveness curve indicates the probability that given the uncertainty in the model inputs, ticagrelor would be cost-effective relative to clopidogrel, for increasing values of willingness to pay for an additional QALY. Based on World Health Organization (WHO) recommendations, a benchmark willingness to pay could be linked to GDP, with 1-3 × GDP per capita representing a reasonable range for an acceptable ICER. ${ }^{(19)}$

\section{RESULTS}

Applying the representative unit costs for Singapore, the total costs for the one-year PLATO study period are presented (Table III). Ticagrelor was associated with lower hospitalisation-related costs when the study drug costs were excluded, as compared to clopidogrel. The cost difference reflected the reduced event rates in the ticagrelor treatment; in particular, ticagrelor treatment was associated with fewer bed days and interventions. These cost savings (SGD 557) partially offset the incremental drug cost (SGD 1,354) of ticagrelor compared to generic clopidogrel, and resulted in an incremental cost (SGD 798) for ticagrelor. The base case lifetime incremental cost for ticagrelor compared to generic clopidogrel was SGD 1,328 and the QALY gain was 0.13. This resulted in a cost per QALY of SGD 10,136 (Table IV). The QALY gain was primarily driven by mortality benefit.

The ICER for ticagrelor vs. clopidogrel was robust over a wide range of input parameters, with changes in most inputs leading to 
Table III. Mean healthcare costs per patient (reflecting the PLATO trial). ${ }^{(8,10)}$

\begin{tabular}{|c|c|c|c|}
\hline Healthcare costs (SGD) & Ticagrelor $(n=5,347)$ & Clopidogrel $(n=5,339)$ & Difference $^{+}(95 \% \mathrm{Cl})$ \\
\hline Total hospitalisation costs & 15,704 & 16,261 & $-557(-1,002$ to -111$)$ \\
\hline Bed-days & 5,256 & 5,526 & $-271(-536$ to -5$)$ \\
\hline Investigations & 2,557 & 2,583 & $-26(-84$ to 31$)$ \\
\hline Interventions & 7,814 & 8,069 & -255 ( -537 to 27$)$ \\
\hline Bleeding-related & 77 & 81 & $-4(-21$ to 11$)$ \\
\hline Study drug cost* & 1,647 & 293 & $1,354(1,331$ to 1,376$)$ \\
\hline Total healthcare costs & 17,350 & 16,553 & 798 (351 to 1,243$)$ \\
\hline
\end{tabular}

* Study drug costs are based on actual utilisation.

${ }^{\dagger}$ Difference is derived from value of ticagrelor subtracted by value of clopidogrel.

Table IV. Results of baseline lifetime cost-effectiveness analysis.

\begin{tabular}{lcccc}
\hline & Ticagrelor & Clopidogrel & Increment & ICER \\
\hline Costs* (SGD) & 27,731 & 26,403 & 1,328 & - \\
$\begin{array}{l}\text { Outcome } \\
\text { Life years }\end{array}$ & & & \\
QALYs & 11.5090 & 11.3568 & 0.1522 & SGD 8,730/life-year \\
\hline
\end{tabular}

${ }^{*}$ For cost-effectiveness analysis, the study drug costs are based on the assumption that future patients would take the drug as prescribed.

QALY: quality adjusted life years; ICER: incremental cost-effectiveness ratio

Table V. One-way sensitivity analysis.

\begin{tabular}{|c|c|c|c|c|c|}
\hline \multirow[t]{2}{*}{ Input parameter } & \multirow[t]{2}{*}{ Baseline value } & \multirow[t]{2}{*}{ Low value } & \multirow[t]{2}{*}{ High value } & \multicolumn{2}{|c|}{ ICER } \\
\hline & & & & Low value & High value \\
\hline Cost of ticagrelor (SGD/day) & 6.00 & 2.00 & 10.00 & Dominant & 21,019 \\
\hline Cost of clopidogrel (SGD/day) & 1.05 & 0.30 & 1.50 & 12,164 & 8,920 \\
\hline Discount rate (\%) & 3.00 & 0.00 & 7.00 & 8,096 & 13,098 \\
\hline Annual risk of $\mathrm{MI}$ in the no event state & 0.019 & 0.010 & 0.038 & 10,113 & 10,114 \\
\hline \multicolumn{6}{|l|}{ Increased risk of death } \\
\hline No event state & 2.00 & 1.00 & 4.00 & 8,296 & 13,694 \\
\hline Nonfatal Ml state & 6.00 & 3.00 & 12.00 & 10,061 & 10,271 \\
\hline Post-MI state & 3.00 & 1.50 & 6.00 & 11,010 & 9,399 \\
\hline Nonfatal stroke state & 7.43 & 3.72 & 14.86 & 10,126 & 10,153 \\
\hline Post-stroke state & 3.00 & 1.50 & 6.00 & 10,010 & 10,259 \\
\hline \multicolumn{6}{|l|}{ Annual cost (SGD) } \\
\hline No event state & 783 & 392 & 1,566 & 9,581 & 11,248 \\
\hline Nonfatal MI state & 6,929 & 3,465 & 13,858 & 10,041 & 10,327 \\
\hline Post-MI state & 783 & 392 & 1,566 & 10,302 & 9,804 \\
\hline Nonfatal stroke state & 9,999 & 5,000 & 19,998 & 10,113 & 10,184 \\
\hline Post-stroke state & 2,161 & 1,081 & 4,322 & 10,042 & 10,325 \\
\hline \multicolumn{6}{|l|}{ Annual QALY weight in nonfatal MI state } \\
\hline Age $<69$ yrs & 0.87 & 0.44 & 1.00 & 13,321 & 9,483 \\
\hline Age $70-79$ yrs & 0.84 & 0.42 & 1.00 & 12,451 & 9,479 \\
\hline Age $>79$ yrs & 0.78 & 0.39 & 1.00 & 10,648 & 9,872 \\
\hline \multicolumn{6}{|l|}{ Annual QALY decrement } \\
\hline Nonfatal MI state & 0.06 & 0.03 & 0.13 & 10,127 & 10,152 \\
\hline Post-MI state & 0.06 & 0.03 & 0.13 & 10,279 & 10,148 \\
\hline Nonfatal stroke state & 0.14 & 0.07 & 0.28 & 10,133 & 10,143 \\
\hline
\end{tabular}

ICER: incremental cost-effectiveness ratio; MI: myocardial infarction; QALY: quality adjusted life years

a change in ICER of no more than SGD 5,000 per QALY (Table V). One exception was drug cost - ticagrelor became dominant (both cost-saving and more effective) when its daily cost approached that of clopidogrel, and the ICER for ticagrelor increased as the cost of clopidogrel decreased. Changes in the other inputs led to plausible movement of the ICERs. For example, since much of the value of ticagrelor appears to be due to a reduction in $\mathrm{MI}$ and death, reducing the utility weight of those states raised the ICER for the drug.

The raw results of the 10,000 bootstrap replications (Fig. 2) indicated that nearly all the simulations fell in the upper right quadrant of the ICER plane, below the threshold line for satisfying the nominal WHO criteria of $1 \times$ GDP per capita. Since this criterion is not uniformly accepted or necessarily appropriate in specific decision-making contexts, the analysis is also presented as a cost-effectiveness acceptability curve (Fig. 3). Here, the probability that ticagrelor is cost-effective, based on the proportion of simulations satisfying a given threshold criterion for willingness to pay, rose as the threshold value increased. Above an acceptable ICER of SGD 15,000, the probability of being cost-effective was approximately $90 \%$; as the threshold increases to the per capita GDP, the probability exceeded 99\%. 


\section{DISCUSSION}

Despite the adoption of contemporary guidelinerecommended therapies, ACS remains a significant cause of death and morbidity in Singapore. The availability of newer therapies demonstrated to be safe and efficacious in large international clinical trials expands the range of therapies available to the treating physician, and could ideally lead to improved overall clinical outcomes. However, newer alternatives are frequently costlier than established generic therapies. Therefore, as new alternatives are introduced, there exists a need to examine not only the comparative effectiveness of therapies available, but also the relative cost-effectiveness of the different therapies so as to maximise the limited and finite healthcare resources available.

In Singapore, no previous study has estimated the relative cost-effectiveness of a drug used to treat ACS prior to the introduction of the drug. The availability of the cost-effectiveness model developed by Nikolic et al afforded us the unique opportunity to perform this pioneering analysis. ${ }^{(10)}$ Above and beyond this, it also provided us the chance to explore the degree to which this model may be applied in local settings. Our study showed that treating a patient with ACS in Singapore for a year with ticagrelor as compared to generic clopidogrel, priced at SGD 6.00 and SGD 1.05, respectively, would be associated with an ICER of approximately SGD 10,000 per QALY, with all the assumptions as outlined previously. This estimate was robust even after performing a series of sensitivity analyses on the basecase scenario.

Singapore has health outcomes comparable with those of developed countries, despite a national healthcare expenditure of about $4 \%$ of GDP, which is considered low among developed countries. ${ }^{(20,21)}$ Universal healthcare coverage is available for all citizens, based essentially on a system of copayment, with different levels of subsidies and assistance schemes. At present, there is no published Singapore guidelines with regard to the level at which a therapy would be considered cost-effective in the local context. As Singapore's GDP per capita is high at nearly SGD 60,000 and the proportion of GDP spent on healthcare is low, the nominal WHO criteria of $1 \times$ GDP per capita may not be applicable in Singapore. Nevertheless, despite accepting a considerably lower societal threshold for willingness to pay, our study indicates that the probability of the proposed strategy of treatment with a year of ticagrelor was likely to be cost-effective.

The degree to which this impacts policy and payer decisions in Singapore is currently minimal. Cost-effectiveness studies are not currently mandated prior to drug approval, and ticagrelor is therefore already approved for use in Singapore as an alternative to clopidogrel in treating ACS. However, it is likely that with soaring healthcare costs and increased patient expectations, Singapore may well require cost-effectiveness data to supplement clinical data prior to licensing approval in the future, as is the case in other countries in the Asia-Pacific region, such as Taiwan

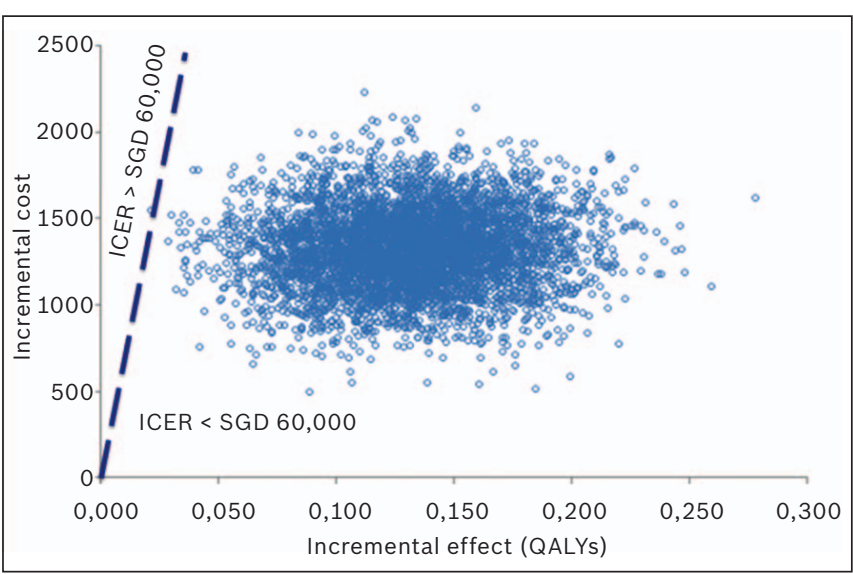

Fig. 2 Probabilistic sensitivity analysis for 10,000 iterations, accounting for variability in trial data and estimates of long-term risk and cost. The dotted line represents the nominal acceptable willingness-to-pay threshold recommended by WHO of $1 \times$ GDP per capita (approximately SGD 60,000).

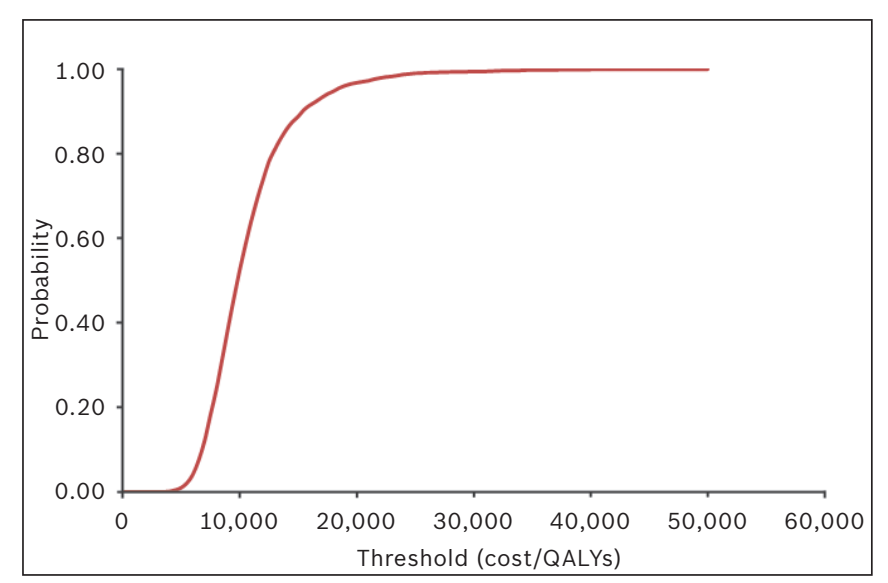

Fig. 3 Cost-effectiveness acceptability curve. Based on 10,000 iterations, the curve represents the estimated probability that ticagrelor would be deemed to be cost-effective at different willingness-to-pay (WTP) threshold levels. For example, if an acceptable cost per additional quality adjusted life year (QALY) is SGD 60,000 (the approximate GDP per capita in Singapore, a common WTP threshold level used by WHO), then ticagrelor has a $99 \%$ probability of being cost-effective. ${ }^{(19)}$

and Korea. Nevertheless, even before that stage, comparative cost-effectiveness analyses such as this may help guide individual hospitals and clinics in Singapore to include the drug in their local formulary.

For the individual treating physician and patient, analyses such as this may help to inform the decision to pay for additional premium for improved outcomes. This will become increasingly pertinent as more new therapies, priced higher than current generic treatments, are introduced into the market. Nevertheless, we have to be prescient and recognise that societal willingness to pay may not necessarily be concordant with the individual's ability to pay.

Finally, our analysis has demonstrated that that the model developed by Nikolic et al can be made applicable from a Singapore healthcare perspective as long as efforts are made to draw on the available data resources. ${ }^{(10)}$ In our analysis, we used data from the local institution level and published government sources, as well as published data from a comparable country. 
Our study has some limitations. First, as already outlined, certain assumptions were made when applying the model to the Singapore context. These assumptions may not necessarily hold true, but the sensitivity analyses performed to test the rigour of the assumptions have shown that our results are robust. Second, the analysis was performed with current costs and hence the ICER may change in the future with changes in costs. Nevertheless, our sensitivity analyses have shown that with the anticipated reduction in ticagrelor price over time, the ICER would be anticipated to reduce. Third, the model assumes that the treatment of patients with ACS in the first year in the local context will be similar to that of patients in the PLATO study. This may not necessarily hold true, as it is recognised that patients in clinical studies frequently have better clinical care and outcomes than 'real-world' patients. Fourth, the model is based on the overall PLATO study results, and hence if clinical events and outcomes of ACS patients in Singapore differ significantly from that of patients in the overall PLATO study, the ICER would also be affected. For instance, a prespecified PLATO subanalysis reported that ticagrelor had less effect in North America as compared to the rest of the world. ${ }^{(22)}$ This may have been due to chance alone, or from an interaction with a higher dose of aspirin used in North America. Currently, it is unknown if such an interaction would apply in Singapore as well, although reassuringly, the use of higher dose aspirin in Singapore is not common and the prescribing information approved by the Singapore Health Sciences Authority clearly recommends that ticagrelor should be used with a low maintenance daily dose of aspirin 75-150 mg. Additionally, the overall higher mortality rates observed in PLATO, as compared to other cardiovascular studies, has been commented on. ${ }^{(23)}$ Although other commentators have found this cross-trials comparison highly problematic due to different trial designs, once again, if 'real-world' outcomes do not mirror that observed in the PLATO study, the cost-effectiveness equation would be affected. ${ }^{(24)}$ Fifth, the side-effects observed more frequently with ticagrelor in PLATO, specifically dyspnoea, may result in increased 'costs' to patients taking ticagrelor due to clinic visits or time off work. While dyspnoea-related hospitalisations have not been separately flagged in the model, the actual bed-day costs for dyspnoea-related hospitalisations are included. As total dyspnoea costs are not separately calculated in the model, the degree to which this may affect the ICER is unknown.

In conclusion, based on PLATO trial data, one-year treatment with the combination of ticagrelor and aspirin versus generic clopidogrel and aspirin in ACS patients, relative to WHO reference standards, is likely to be cost-effective from a Singapore public healthcare perspective.

\section{ACKNOWLEDGEMENTS}

The authors would like to thank Malcolm Koh and Zhang Jing for their assistance in identifying the unit costs used in the manuscript. They did not receive compensation for their assistance, apart from their employment at the institution where the study was conducted.

\section{CONFLICTS OF INTEREST}

Mellstrom C is an employee of AstraZeneca. Chin CT's institution has received honoraria from AstraZeneca for his involvement in the study - research grant from AstraZeneca. Matchar DB has received consultant fees from AstraZeneca - honoraria from AstraZeneca. Chua TS has no conflict of interest to declare.

\section{FUNDING SOURCES}

This work was supported by AstraZeneca. Academic members of the PLATO Executive Committee designed the PLATO trial in collaboration with representatives from AstraZeneca. Results were interpreted by the authors, with representatives from the sponsor.

\section{REFERENCES}

1. National Registry of Diseases. Information paper on acute myocardial infarction in Singapore, 2007-2008. Available at: http://www.nrdo.gov. sg/uploadedFiles/NRDO/Publications/20110211 AMI Information Paper 2010 v3.pdf. Accessed January 7, 2013.

2. Chan MY, Shah BR, Gao F, et al. Recalibration of the Global Registry of Acute Coronary Events risk score in a multiethnic Asian population. Am Heart J 2011; 162:291-9.

3. 2012 Writing Committee Members, Jneid H, Anderson JL, et al. 2012 ACCF/ $\mathrm{AHA}$ focused update of the guideline for the management of patients with unstable angina/Non-ST-elevation myocardial infarction (updating the 2007 guideline and replacing the 2011 focused update): a report of the American College of Cardiology Foundation/American Heart Association Task Force on practice guidelines. Circulation 2012; 126:875-910.

4. Kushner FG, Hand M, Smith SC Jr, et al. 2009 Focused Updates: ACC/AHA Guidelines for the Management of Patients With ST-Elevation Myocardial Infarction (updating the 2004 Guideline and 2007 Focused Update) and ACC/AHA/SCAI Guidelines on Percutaneous Coronary Intervention (updating the 2005 Guideline and 2007 Focused Update): a report of the American College of Cardiology Foundation/American Heart Association Task Force on Practice Guidelines. Circulation 2009; 120:2271-306.

5. Hamm CW, Bassand JP, Agewall S, et al. ESC Guidelines for the management of acute coronary syndromes in patients presenting without persistent ST-segment elevation: The Task Force for the management of acute coronary syndromes (ACS) in patients presenting without persistent ST-segment elevation of the European Society of Cardiology (ESC). Eur Heart J 2011; 32:2999-3054.

6. Steg PG, James SK, Atar D, et al. ESC Guidelines for the management of acute myocardial infarction in patients presenting with ST-segment elevation: The Task Force on the management of ST-segment elevation acute myocardial infarction of the European Society of Cardiology (ESC). Eur Heart J 2012; 33:2569-619.

7. Husted S, Emanuelsson H, Heptinstall S, et al. Pharmacodynamics, pharmacokinetics, and safety of the oral reversible P2Y12 antagonist AZD6140 with aspirin in patients with atherosclerosis: a double-blind comparison to clopidogrel with aspirin. Eur Heart J 2006; 27:1038-47.

8. James $\mathrm{S}$, Akerblom A, Cannon $\mathrm{CP}$, et al. Comparison of ticagrelor, the first reversible oral P2Y(12) receptor antagonist, with clopidogrel in patients with acute coronary syndromes: Rationale, design, and baseline characteristics of the PLATelet inhibition and patient Outcomes (PLATO) trial. Am Heart J 2009; 157:599-605.

9. Wallentin L, Becker RC, Budaj A, et al. Ticagrelor versus clopidogrel in patients with acute coronary syndromes. N Engl J Med 2009; 361:1045-57.

10. Nikolic E, Janzon M, Hauch O, et al. Cost-effectiveness of treating acute coronary syndrome patients with ticagrelor for 12 months: results from the PLATO study. Eur Heart J 2013; 34:220-8. Epub June 19, 2012.

11. Norhammar A, Stenestrand U, Lindback J, Wallentin L. Women younger than 65 years with diabetes mellitus are a high-risk group after myocardial 
infarction: a report from the Swedish Register of Information and Knowledge about Swedish Heart Intensive Care Admission (RIKS-HIA). Heart 2008; 94:1565-70.

12. Dennis MS, Burn JP, Sandercock PA, et al. Longterm survival after first-ever stroke: the Oxfordshire Community StrokeProject. Stroke 1993; 24:796-800.

13. Olai L, Omne-Ponten M, Borgquist L, Svardsudd K. Survival, hazard function for a new event, and healthcare utilization among stroke patients over 65 years old. Stroke 2009; 40:3585-90.

14. Ministry of Health, Singapore. Hospital Bill Sizes. Available at: http:// www.moh.gov.sg/content/moh_web/home/costs_and_financing/ HospitalBillSize.html. Accessed January 7, 2013.

15. Management Center for Health Promotion, Republic of Korea. Costeffectiveness analysis of national prevention programs for cardiovascular disease, 2007.

16. Burström K, Johannesson M, Rehnberg C. Deteriorating health status in Stockholm 1998-2002: results from repeated population surveys using the EQ-5D. Qual Life Res 2007;16:1547-53.

17. Department of Statistics, Singapore. Complete Life Tables 2006-2011 for Singapore Resident Population. Available at: http://www.singstat.gov.sg/ pubn/popn/lifetable06-11.pdf. Accessed January 7, 2013.

18. Gold MR, Patrick DL, Torrance GW, et al. Identifying and valuing outcomes.
In: Gold MR, Siegel JE, Russell LB, Weinstein MC, eds. Cost-Effectiveness in Health and Medicine. New York: Oxford University Press, 1996.

19. Sachs JD. Macroeconomics and Health: Investing in Health for Economic Development. Report of the Commision on Macroeconomics and Health. Geneva, Switzerland: World Health Organisation, 2001. Available at: http:// whqlibdoc.who.int/publications/2001/924154550x.pdf. Accessed January 7, 2013.

20. World Health Organization. Global Health Observatory Data Repository. Available at: http://apps.who.int/ghodata/?theme=country. Accessed January 7, 2013.

21. Ministry of Health, Singapore. Costs and Financing. Available at: http:// www.moh.gov.sg/content/moh_web/home/costs_and_financing.html. Accessed January 7, 2013.

22. Mahaffey KW, Wojdyla DM, Carroll K, et al. Ticagrelor compared with clopidogrel by geographic region in the Platelet Inhibition and Patient Outcomes (PLATO) trial. Circulation 2011; 124:544-54.

23. Serebruany VL. Viewpoint: paradoxical excess mortality in the PLATO trial should be independently verified. Thromb Haemost 2011; 105:752-9.

24. Ohman EM, Roe MT. Explaining the unexpected: insights from the PLATelet inhibition and clinical Outcomes (PLATO) trial comparing ticagrelor and clopidogrel (editorial). Thromb Haemost 2011; 105:763-5. 